\title{
Bengt Strömgren's Approach to the Galaxy
}

\author{
Bengt Gustafsson \\ ${ }^{1}$ Department of Physics \& Astronomy, Uppsala University, \\ Box 515, SE-75120 Uppsala, Sweden \\ email: bg@astro.uu.se
}

\begin{abstract}
The contributions of Bengt Strömgren to the exploration and understanding of the Galactic Disk are sketched. The question what we can learn from his systematic approach is discussed.
\end{abstract}

Keywords. Galaxy: disk; stars: fundamental parameters; techniques: photometric; orbituaries, biographies; history and philosophy of astronomy

\section{A background}

To celebrate the 100th anniversary of Bengt Strömgren's birthday is also to celebrate one century of astrophysics, with remarkable scientific progress. In his lifetime achievement, Bengt Strömgren embodies much of this development. Early well schooled in classical astronomy and heavy numerical calculations, he was exposed to the birth of quantum mechanics and modern atomic physics. This made him contribute fundamentally to stellar and interstellar-matter physics. Later, he added to this new developments in photoelectric stellar photometry, and thus created of these different components a fundamental basis for Galactic exploration. Many contemporary important results in this exploration can be directly traced back to Strömgren's suggestions and inspiration. For this reason, the topic of the present symposium is a natural choice in this year.

\section{The early years}

Strömgren was born into astronomy in 1908 and achieved, partly through his father who was astronomy professor Elis Strömgren in Copenhagen, a flying start in research with his first published papers (reports on observations of comet Baade) at an age of 14. (For a detailed account of Bengt Strömgren's early career, see Rebsdorf 2005.) He got engaged in an astrometric programme at the transit instrument of the Observatory at Øster Voldgade, resulting in a catalogue of $\alpha$ and $\delta$ for 131 northern bright stars. This work was followed by papers on Die Bestimmung erster parabolischer Bahnen unter Anwendung der Rechenmaschine from 1925, which was the beginning of his doctoral thesis work. He also published early work on photoelectric registration of stellar meridian transits and on numerical calculations of perturbations of asteroid orbits.

Strömgren got familiar with various aspects of classical astronomy, but also personally familiar with leading astronomers, not the least through his father who had many international contacts. And, then the world was small! Later, in 1932 when Strömgren entered the International Astronomical Union, there were totally about 400 members of the organization. When he left the position as its Secretary General in 1952, this number had increased by about a factor of 1.6, and when he finished as its President in 1973, it had increased by a factor of 5, up to 2000. Today the number is again increased by a factor of 5 , close to 10,000 . So, the world was different, and it was possible to know most important astronomers. So did Bengt, and this was eased by his social gifts, his profound 


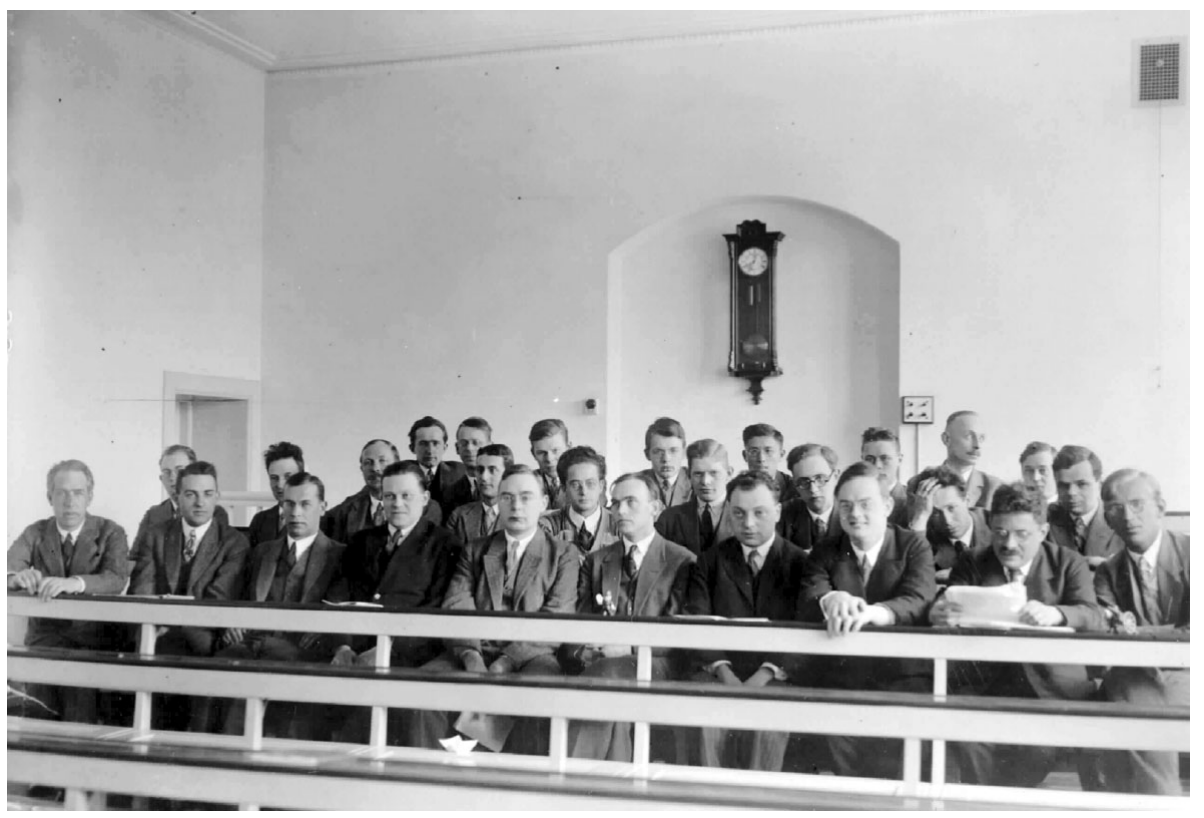

Figure 1. Participants at the Copenhagen Conference in 1929 in Auditorium A the present Niels Bohr Institute. Front row, from left: N. Bohr, R. Kronig, I. Waller, J. Holtsmark, H. Kramers, S. Rosseland, W. Pauli, E. Jordan, P. Ehrenfest and G. Gamow. Second row: L. Rosenfeld (no. 1 from left), O. Klein (2), S. Goudsmit (4), C. Møller (5) and W. Heitler (8). Third row, from left: B. Strömgren (3) and H. Casimir (4). Photo: Niels Bohr Archive.

curiosity concerning what other people were doing, and his extraordinarily good memory, not only for science but also for people.

Strömgren, however, also early got in touch with another, maybe even more important circle: the extensive group of young physicists around Niels Bohr at the newly founded Institute for Theoretical Physics of Copenhagen University, later named after Bohr. There, during the inspiring youth of Quantum Mechanics, Strömgren met a number of leading atomic physicists, among whom, in addition to Niels Bohr, he considered Oskar Klein and the guests Ralph Howard Fowler, Cecilia Payne and Friedrich Hund to be particularly important for him (Strömgren 1983). He got interested in applying the new physics to stellar spectra and stellar modelling in general, and thus turned from a classical astronomer to a modern astrophysicist. He began work on stellar structure, contributed to solving what Arthur Eddington had named "the opacity discrepancy" by computing actual opacity tables, and showed that one could not avoid the conclusion that the Sun and stars contained much more hydrogen than Eddington had assumed earlier (Strömgren 1932). This demonstrates a characteristic feature of Strömgren's theoretical work through the years: he did not only develop the theory but also followed it up with detailed numerical calculations to make the theoretical advances directly applicable.

\section{Physics of stars and HII regions}

Bengt Strömgren was invited in 1936 by Otto Struve to an assistant professorship at the University of Chicago and Yerkes and McDonald Observatories. Here, he had detailed and continuing discussions with Subrahmanyan Chandrasekhar on problems of stellar structure, and discussed with William W Morgan on spectral classification - Morgan was close to finishing his and Philip Keenan's new two-dimensional classification of stellar 


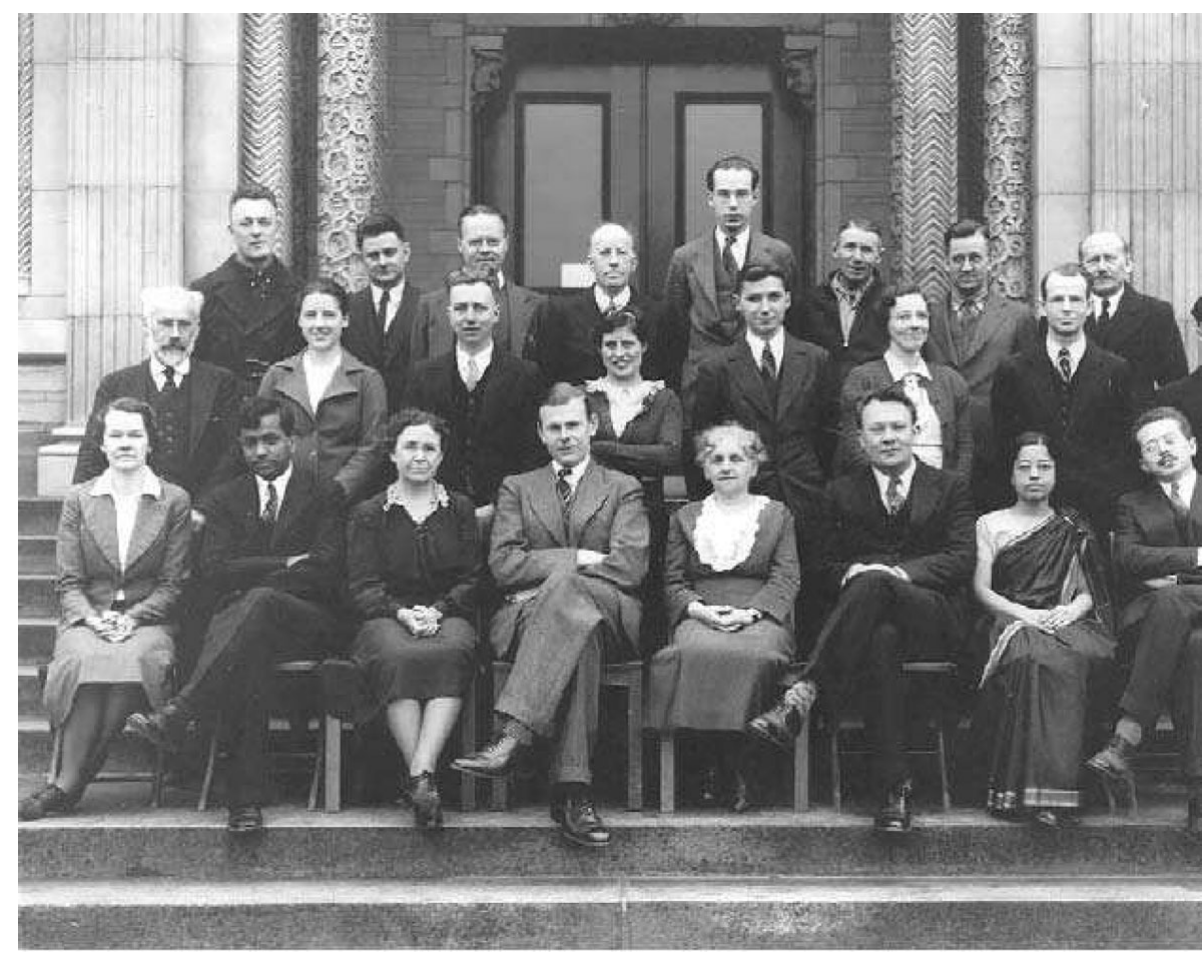

Figure 2. Yerkes Observatory staff in 1936 or 1937. First row, from left: S. Chandrasekhar (no. 2), B. Strömgren (4), G. P. Kuiper (6) and J. Greenstein (8). In second row: G. van Biesbroeck (1) and W.W. Morgan (7). In third row: O. Struve (3). Photo: Yerkes Observatory.

spectra, the MK classification. Strömgren also followed up Otto Struve's discovery of extensive areas in the Milky Way that emitted faintly in the hydrogen Balmer lines, and showed that if a hot early-type star is imbedded in a thin interstellar hydrogen cloud, the transition zone between the ionized gas close to the star and the more distant gas that remains neutral is quite narrow (Strömgren 1939). This research led to one of the three astronomical concepts that carry his name, the Strömgren sphere (the other two being an asteroid and his photometric system). After 18 months in the United States Strömgren returned to Copenhagen to take up a full professorship there, and soon also the directorship of the Copenhagen Observatory after his father.

Strömgren now turned to the solar atmosphere, including the opacity of the negative hydrogen ion, which Rupert Wildt had just found to be significant. Strömgren made a pioneering first model-atmosphere analysis of the solar spectrum and published the result in a Festschrift for his father's 70th birthday (Strömgren 1940). The values of chemical abundances derived agree astonishingly well with present values, within a factor of two for the elements studied. By this work he set a standard for quantitative analysis of stellar spectra, which was inspiringly high. In fact, when he summarised the stellar-atmosphere field at a conference (Strömgren 1955), he had the optimistic vision that an accuracy of one percent should be obtainable in predicted continuous fluxes and line profiles from model atmospheres. Not until recently, half a century later, has that goal been reached, and yet not for all types of stars and all spectral lines.

During the war in isolated Copenhagen, Strömgren took interest in geometrical optics and devised an original Schmidt refractor and calculated optical sine tables, both 
contributions that he was proud of. After the war he built up an astrophysics school in Copenhagen with young scientists like Jean Claude Pecker, Évry Schatzman and Anne Underhill. He also spent periods in the USA, and finally in 1950 he left Copenhagen to become professor at the University of Chicago and director of Yerkes and McDonald Observatories. It was during these tours to the USA that his work on the evolution of the Galaxy definitely began, with the development of the photometric system, the uvby $\beta$ photometry that is often called Strömgren photometry.

\section{The new tools}

The dominant kind of detectors used in astronomical photometry during the first half of the century were the photographic plates. Their low efficiency and non-linear response limited the accuracy of the resulting measures. Strömgren saw early new possibilities open up, which would finally drastically change this situation. As he has told in his autobiographical notes (Strömgren 1983), 14 years old he visited Paul Guthnick, at the Berlin-Babelsberg Observatory, who was pioneering photoelectric photometry with a 30 $\mathrm{cm}$ refractor equipped with a gas-filled photocell with a potassium cathod. In the early forties Strömgren experimented himself with electrometer vacuum tubes "and was partly successful". He also elaborated various aspects of stellar photometry in two chapters in Handbuch der Experimentalphysik (Strömgren 1937) and demonstrated the advantages of filters that were narrower than the traditional broad-band colour filters. In particular, the errors caused by extinction, both interstellar and terrestrial, due to the shift of the effective wavelength of the filters with changing stellar flux distributions, would limit the accuracy at high-precision work.

However, neither did the photographic plates enable such accuracy, nor were such narrow filters accessible. But after the war the situation was totally changed. The photomultiplier tube with its relatively high sensitivity and linear response, though invented already in the thirties, had now been applied to astronomy by Joel Stebbins and Albert Whitford, and their younger collaborators Gerald Kron, Olin Eggen, and Harold Johnson. Strömgren got acquainted with them and proceeded in 1948 at the McDonald Observatory to use the technique to measure the $\mathrm{H} \beta$ strength in late- $\mathrm{A}$ and early $\mathrm{F}$ stars through interference filters, which had also now become available. He used two bands, both about $200 \AA$ wide, one across the hydrogen line, and one beside it. He reported to the IAU General Assembly in 1948: "Very promising results have been obtained by the use of interference filters and photoelectric photometry for spectral classification.... a mean error corresponding to $0^{\mathrm{m}} .006$ (one observation) was determined. It follows that spectral classification...can be effected with an accuracy of, for instance, better than one-tenth of a spectral class in the spectral ranges A5 to F0" (Strömgren 1950). Later, when narrower interference filters were available, he placed one $30 \AA$ band on the line, and two broader comparison bands beside it, in defining what he called the $l$ index (Strömgren 1954). Finally, some years later the Crawford-Strömgren $\mathrm{H} \beta$ index was devised with one even narrower and one broad band, both centred on the line.

It is noteworthy, that Strömgren's successful first demonstration of the photoelectric technique for quantitative classification seems to have been a by-product of an attempt to survey interstellar Balmer emission. In general, his different studies in various fields were often linked in a logical and systematic way. However, Strömgren later explicitly ascribed his interest in accurate stellar photometry to his experience, through his model atmosphere work, that hydrogen criteria were indeed the most reliable indicators for temperature and surface gravity for stars hotter than the Sun (Strömgren 1983). Also, the previous photographic stellar spectro-photometry by Daniel Barbier and Daniel Chalonge 
(1939), by Bertil Lindblad (1922) and Yngve Öhman (1935), as well as by Robert M. Petrie (Petrie \& Maunsell 1949), inspired him to measure the Balmer lines in stellar spectra. These sources of inspiration were obviously significant also for his following attempts to measure the Balmer discontinuity photoelectrically. The first step, which he called the $c$ index, and which was later developed into the $c_{1}$ index in the uvby system, was thus established by 1951 (Strömgren 1951a). With his background he found it important to position the ultraviolet $u$ band of the new system with its transmission entirely at shorter wavelengths than the Balmer discontinuity, unlike the location of the $U$ band in the pioneering Six-colour photometry of Stebbins \& Whitford (1943) or the corresponding band in the broad-band $U B V$ system (Johnson \& Morgan 1951), which was established in parallel with Strömgren's work at Yerkes and McDonald observatories. In addition, already at this stage Strömgren set out to explore the possibilities to establish a two-dimensional classification for the late-type stars, from F8 to K6, by photoelectric photometry together with Kjeld Gyldenkerne (see also Strömgren \& Gyldenkerne 1955). Also here, the inspiration came from earlier photographic work in Uppsala and Stockholm by Bertil Lindblad and collaborators (see Lindblad \& Stenquist, 1934, and references therein). Strömgren enthusiastically reported probable errors in this spectral classification "of two to four hundreds of a spectral class". Finally, this effort was to lead to the Brorfelde or gnkmf system, and also inspired other narrow-band systems like the DDO system and the Vilnius system.

Strömgren had with his $l$ - $c$ system devised a method for two-dimensional quantitative spectral classification which could provide estimates of stellar temperature and luminosity or surface gravity, a system which would soon be shown to be the most accurate method for stars in the spectral interval B-F. However, in the early fifties a very important discovery was made by Joseph Chamberlain and Lawrence Aller (1951). They analysed spectra the two subdwarfs HD 19445 and HD 140283 and proved them to be significantly metal poor, by a factor of ten or even more as compared with the Sun. This was immediately recognized to be a strong indication that the heavy elements were, indeed, made in stars, and that the gradual build-up of these elements could be traced by measuring the metallicities of stars of various ages and populations. Studying stars of different metallicity should be a way of studying the history of our Galaxy. A photometric system for such purposes obviously also needed a way of estimating metallicity. In order to measure the metallicities quantitatively, Strömgren added an $m$ index to his photometric system. It had three bands with half widths of about $90 \AA$, one across a region with strong metal lines at $4030 \AA$, one at $4500 \AA$ and one at $5000 \AA$, and the index was defined as $m=$ constant $-2.5[\log I(5000)+\log I(4030)-2 \log I(4500)]$. Three years later, Nancy Roman had demonstrated the existence of a group of F stars with high spatial velocities and very weak metal lines (Roman 1954). Strömgren could show that Nancy Roman's stars stood out from the typical F stars by about 0.1 magnitudes in the $m$ index, more than 10 times the observational error in $m$, and that the index value correlated with the ultraviolet excess $\delta(U-B)$ which was thought to be a metallicity indicator (Strömgren 1958a).

\section{The populations}

In May 1957, a conference on Stellar Populations was arranged at the Pontifical Academy of Science in the Vatican. This conference turned out to be one of the important ones in the history of 20th century astronomy. The participants were about 20 of the leading astronomers, including Walter Baade, Adrian Blaauw, William Fowler, George Herbig, Fred Hoyle, Georges Lemaître, Bertil Lindblad, W.W. Morgan, Edwin Salpeter, 


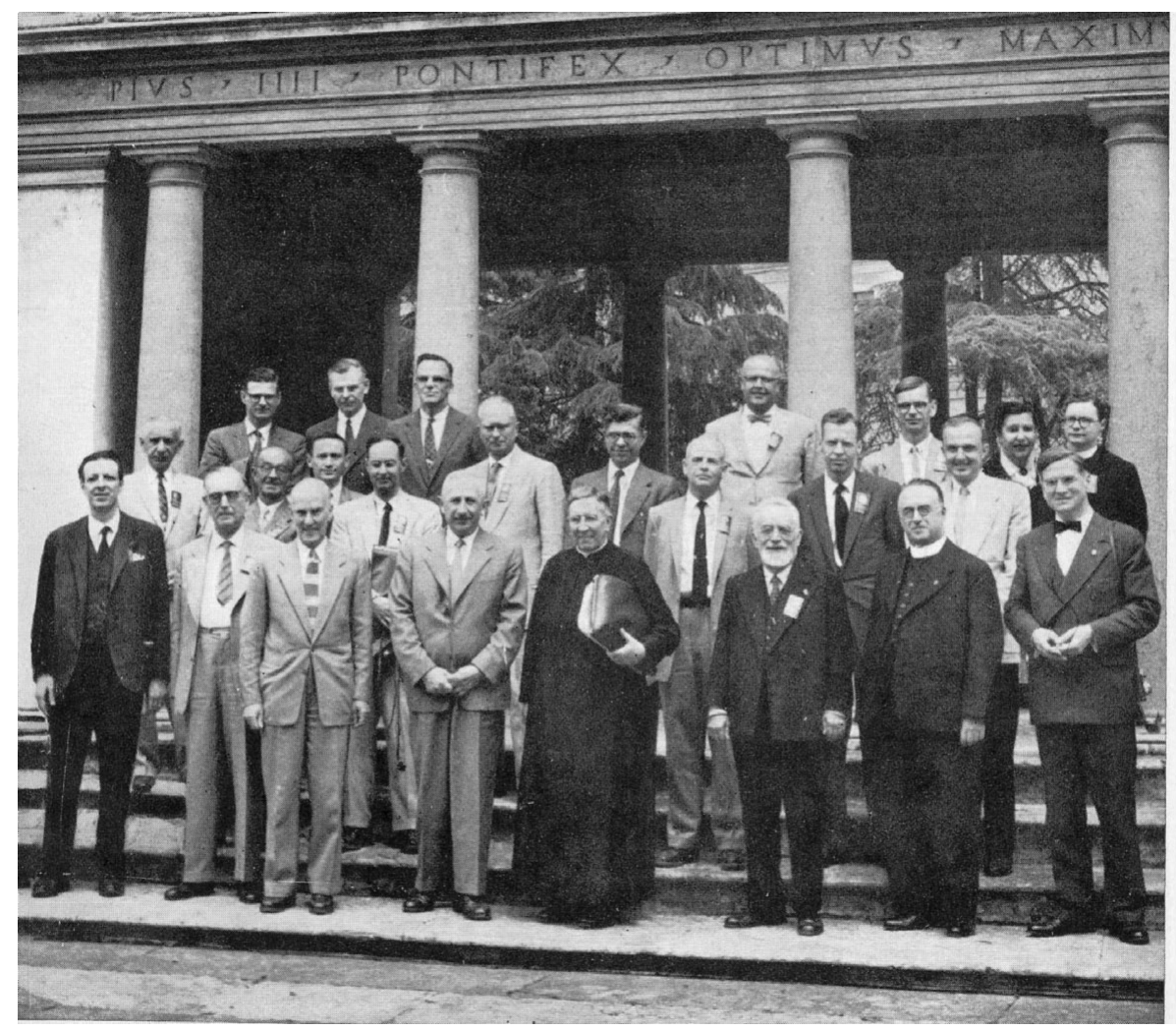

Figure 3. Participants at the Vatican Conference on Stellar Populations in May 1957. First row, from left: P. Salviucci, B. Lindblad, J.H. Oort, W. Baade, D. O'Connell, G. Armellini, G. Lemaitre, H.A. Brueck. Second row: J.J. Nassau, D. Chalonge, M. Schwarzschild, A. Blaauw, O. Heckmann, F. Hoyle, W.W. Morgan, G.H. Herbig, A.R. Sandage. Third row: E.E. Salpeter, B. Strömgren, L. Spitzer, W.A. Fowler, A.D. Thackeray, V. Preobrajenski, P. Treanor. Photo: Specola Vaticana.

Allan Sandage, Martin Schwarzschild, Lyman Spitzer and Bengt Strömgren. Strömgren presented two papers, one on the composition differences between stellar populations, based on his $m$-index measurements (Strömgren 1958a), and one on spectrophotometric classification of the population groups, where also the power of the $l$ and $c$ indices was demonstrated (Strömgren 1958b). It is greatly interesting to now, half a century later, read the well minuted discussions at this symposium (O'Connell 1958). Although the concept of stellar populations in the Galaxy had been introduced by Baade already in 1944 in his studies of the stellar content in the Andromeda galaxy and its companion galaxies (Baade 1944), and in fact hinted at by Oort (1926) and Lindblad (1925, 1926, 1927) almost two decades before on kinematical grounds, the full complexity of the concept and its significance for the evolution of the Galaxy were now established at the conference. This was in particular discussed in a presentation at the Symposium by Jan Oort (Oort 1958), who distinguished five different stellar populations: Halo population II, Intermediate population II, Disk population, Intermediate population I and Extreme population I with different characteristics. A great number of questions concerning the origin and interrelations between these various populations were discussed but remained unanswered. A discussion of this conference and its importance for the 
population concept was presented by Blaauw at the IAU Symp. 164 (Blaauw 1995). The Vatican conference formed a significant basis for Strömgren's continued efforts to study and map the evolution of the Galaxy.

\section{The programme}

It is clear that Strömgren already the following year had essential parts of his programme ready for Galactic stellar astronomy. In his Halley lecture from 1958 (Strömgren 1958c) he reported about the potential of his $l c$ system: "It would seem that photoelectric narrow-band photometry can lead to information on the initial composition of stars and their ages which may help our efforts to form a picture of the evolution of the Galaxy." He presents the basics of his methods and states, boldly, for B stars: "It is now a simple matter to derive the age of a star from observed values of $l$ and $c$ ". For $\mathrm{F}$ stars, he claims that ages to a relative accuracy of $10-30 \%$ should be possible to obtain: "... such age determinations may prove to be of value. No theoretical massage calibration of the $c-l$ diagram for Population I F stars has as yet been carried out, but it is quite feasible to do so". At this stage Strömgren obviously realized that his accurate photometry offered possibilities to measure the location of individual stars within the main-sequence band, which would enable age determinations, and to determine metallicities with relative errors significantly less than a factor of two. This latter accuracy was fully comparable with what one could obtain for (only) bright stars with high-resolution spectrometers and photographic detectors. Not until the development of echelle spectrometers and electronic detector arrays in the seventies could significantly more accurate metallicity determinations be made.

The importance and the possibilities of these achievements within "quantitative spectral classification" were certainly not yet fully understood in the astronomical community. And a number of efforts were needed before the great questions could be attacked. The photometric system had to be optimized for its purpose. Standard stars had to be established, and the system should be tried out and calibrated, and its potential should be explored and verified. Telescope and photometers should be developed for effective surveys. This required hard work and could not be a single man's effort. Collaborators were needed and this required schooling and inspiration, leadership and organization. And, to use a modern word, selling of the ideas, for instance by picking some striking examples demonstrating the power of the approach.

First, the uvby system had to be finally established, with optimum choices of wavelength bands and observations of standards. This was accomplished by 1960 by observation runs at Lick and Palomar observatories, together with Charles Perry and Robert Cameron. The $\mathrm{H} \beta$ index was developed at Kitt Peak by David Crawford, and there Strömgren also continued his uvby observations.

Next, the system and its possibilities had to be presented to a broad community. This was done in reviews at symposia, and in prize lectures like the Halley Lecture from 1958 and the George Darwin Lecture (Strömgren 1963a). However, descriptions detailed enough to serve as introductions and even "manuals" for future users of the system, including students, were also needed. Strömgren provided two such descriptions, one in the monograph series Stars and stellar systems (Strömgren 1963b) and one particularly important in the then relatively new series of Annual Reviews of Astronomy 83 Astrophysics (Strömgren 1966b). 


\section{Birth places and worries about metallicity}

The key aim of the new system was thus to determine stellar fundamental parameters, effective temperature, surface acceleration of gravity and metallicity, and determine them well enough to be able to deduce stellar ages and masses by comparison to evolutionary tracks. In the mid sixties Strömgren had demonstrated in practice that this was indeed possible, for B, A, and F stars in the main-sequence band. He showed that he was able to obtain an accuracy better than $10 \%$ in stellar age, at least on a relative scale (Strömgren 1966a, Kelsall and Strömgren 1965). It is characteristic that this work was based, in addition to Strömgren's photometry, on stellar evolution calculations by Thomas Kelsall in collaboration with himself; he was anxious to have full insight into all critical aspects in his work, and he was certainly competent enough in stellar modelling and its physical and numerical aspects for this to be possible.

For the younger stars he was able to calculate their orbits backwards in time and thus demonstrate that their places of birth were located in spiral patterns according to the $\mathrm{C}$ C Lin spiral-arm theory. He reported to the IAU Symposim 31 in 1967: "For the 52 stars of the sample we computed the places of formation, using the Galactic gravitational field adopted by Contopoulos and Strömgren (1965) in their calculation of tables of plane Galactic orbits.... The results... do suggest that the majority of the stars considered were formed in our own spiral arm, and that about 10 to $15 \%$ of the stars originated 2 or $3 \mathrm{kpc}$ further away from the Galactic center, i.e. in the Perseus Arm. There is in the sample discussed no evidence for a component contributed by star formation in an inner arm" (Strömgren 1967). Although one could feel that Strömgren sometimes was too optimistic about the accuracy of his results, this was an impressive demonstration of the power of the method. It is also noteworthy that Strömgren had again found a leading specialist, G. Contopoulos in Galactic dynamics, to join in the efforts, but not left the work to calculate orbits to him alone.

For the metallicities based on the $m_{1}$ index there was a worry, raised by Bashek (1960), Conti \& Deutsch (1966) and others, that the majority of the metal lines affecting the $v$ band were located on the flat part of the curve of growth, and thus sensitive to the microturbulence parameter more than to the metal content of the atmospheres. The issue was then whether the microturbulence parameter varied independently of the fundamental parameters of the stars - effective temperature, surface gravity and metallicity (or, more fundamentally, mass, age, and chemical composition). Strömgren found that the tight correlation between the departure of his observed $m_{1}$ from the standard sequence, $\Delta m_{1}$, and spectroscopic $[\mathrm{Fe} / \mathrm{H}]$ values indicated that the effects of additional microturbulence variations were minor (Strömgren 1966b). A decade later, this view was confirmed by spectrophotometry at high resolution by Poul Erik Nissen and others.

Yet, it must be admitted that the $m_{1}$ index was a weak point of the photometric system. Both the Balmer $\delta$ line and the blue bands of the Violet CN system are located in the $v$ band, and affect the index for the hotter and cooler stars. More serious is its relatively low sensitivity to metallicity. The alternative to use bands with weaker but more sensitive spectral lines on the linear part of the Curve of Growth does not exist at this spectral resolution for solar-type stars; such methods were, however, later exploited at higher spectral resolution by Nissen, Kjærgaard and others. However, the possibility to go somewhat towards the ultraviolet in the spectrum and then to also include the wings of strong lines like the Ca II H and $\mathrm{K}$ lines, as is presently tried with the $v$ band of the SkyMapper project (Murphy et al. 2008), could have been attractive. However, such a bandpass would have mixed the abundances of the $\alpha$ element $\mathrm{Ca}$ and Fe. Since these abundances have later been found to vary relative to each other for various stellar 
populations, that might have led to some confusion when uvby photometry was used for defining samples of stars.

\section{The large-scale studies}

Strömgren returned to Copenhagen University and Nordita, the Nordic Institute for Theoretical Physics located at the Niels Bohr Institute, in 1967. He systematically pursued his efforts in Galactic exploration by means of his photometric system. New efficient four-channel photometers were built, a Danish $50 \mathrm{~cm}$ and a $1.5 \mathrm{~m}$ telescope were erected at ESO on La Silla and used a lot for photometry. He engaged a number of young collaborators, like Erik Heyn Olsen, Bent Grønbech and Jens Knude in photometric work, and Poul Erik Nissen in calibrating his photometry by measuring accurate spectroscopic metallicities. He was systematically investigating the potential of his system. When I came to Nordita as a fellow in 1968, Strömgren suggested to me to study the properties of the uvby system for anlysing integrated light from galaxies, with the aim of characterising their stellar population, and in particular to study the possibilities to resolve what was later called the age-metallicity degeneracy in population synthesis. He was also anxious to devise criteria to be able to single out horizontal-branch stars of intermediate population II; he thought of stars like those on the stubby red horizontal branch of the metal-rich globular cluster 47 Tuc, such stars in the Galactic field should be excellent tracers for that population. This required model predictions, and that led me into his old field of stellar model atmospheres.

It should also be mentioned that Strömgren contributed excellent lecture series during these years, both at Nordita/Niels Bohr Institute in its classical Auditorium A, and at the Astronomical Observatory. At the first place he gave series of lectures on stellar evolution and nucleosynthesis, on Galactic dynamics, and also a series on galaxies. These lectures, followed by a wide audience of physicists and astronomers, were dealing with the present research developments and contained excellent summaries and comments on disputed issues. In this art of judging and summarizing recent developments, Strömgren was a true master. These lectures also often reflected Strömgren's own deep research experience. The lecture series given at the Observatory were more operational, dealing with topics like spectrosopy, photometry, and stellar atmospheres. These were very useful as a research education, and in particular valuable for those who were to continue projects along the lines of Strömgren's large programme. It would, in fact, be reasonable to see these lectures as part of the programme itself.

For the F- and early G-type stars, Strömgren was now eager to obtain the distributions of stars on different metallicity within well-defined volumes of space, and to have enough stars to be able to subdivide them into different age bins and space velocities (Strömgren 1969). He accurately mapped the zero-age main sequences of different rich open clusters in order to trace possible differences in helium abundances (Strömgren et al. 1982). And he investigated the distribution of interstellar dust in the solar vicinity by accurately determining the colour excesses of the F stars (Strömgren 1972). These studies had been pioneered by Strömgren earlier but were now, during the seventies and eighties pursued further by his younger collaborators together with him.

It is interesting to see how Strömgren in his late papers, at an age above 75 , still worked towards and stressed his great vision to empirically trace the Galactic populations, and their origins and interrelations. He was particularly intrigued by the discovery by Gilmore and Reid (1983) of a "thick disk", with a scale height of more than 1000 pc, as compared with the standard disk with its scale height of about $300 \mathrm{pc}$, and comprising about $2 \%$ of stars in the solar neighbourhood. The physical character of this population, and its 
relation to "his own" Intermediate Population II, challenged him. In his contribution to IAU Symposium 106, The Milky Way Galaxy (Strömgren 1985), he discussed no less than 7 ongoing studies, of which he was involved in most, that were directed towards the clarification of the histories of the Galactic populations. Among these were the photometric surveys of Erik Heyn Olsen, the radial-velocity survey of Johannes Andersen, Michel Mayor and Birgitta Nordstöm, Jens Knude's studies towards the North Galactic pole, and Torben B Andersen's towards the South polar cap. In other late papers he commented on ongoing spectroscopic work by Nissen and collaborators, as well as by Bengt Edvardsson et al., which can also be seen as part of his grand programme.

It was moving to meet him in these years and note his eagerness to learn about the results as soon as they started appearing; a feeling that one is reminded of when reading his late papers reflecting his curiosity, and his understanding that time was now limited. A typical sentence from this time, taken from one of his very last papers (Strömgren 1987), is: "However, as emphasized... a further strengthening of the data base and a revision of the age scale for intermediate population II stars is required before more definitive conclusions can be drawn. We have referred above to the observational and theoretical work now in progress, which within one or two years should lead to the desired clarification." We, who were engaged in this "work now in progress" know that it took longer time, that the picture got even more complex, and that Strömgren, as much as he would have enjoyed seeing the results, would have appreciated the growing complexity.

\section{What can we learn?}

What can we learn from Strömgren's example, and in particular his approach towards understanding the Galactic disk? In considering this question I first wish to cite Johannes Andersen, who has made the following important remark: "His achievements were so logically designed and systematically pursued that it appeared all natural and self evident to a later generation. How could it be otherwise? Yet, it was otherwise until he showed the way."

Andersen stressed Strömgren's logical and systematic approach. Strömgren contemplated what to do, and did it in the right order and right way. In his teaching, he required us to answer a number of questions in the right order: "What do we wish to observe? Why do we observe this? How do we observe, what means do we use? And Where and When do we do this?" Answer the questions is this order, not, as is often done in practice, in the converse order! And even more important, carry out your project according to a systematic plan, which may well take considerable time. His programme was not so rigid that he did not modify it when new discoveries or experiences so required, but he did not redirect the basic aims, nor did he divert from his main track to follow up other sudden ideas, except for some short excursions.

Second, Strömgren through his example and also in his teaching underlined the importance of being methodologically advanced, and to master your tools. This entailed a considerable interest in details. One example, just out of my own recollection; others at this meeting can give many more: When I decided to start calculating blanketed and convective model atmospheres I needed a flexible and rapid method for radiative transfer with scattering. I had happened to come across Feautrier's new method just developed in the Annales d'astrophysique, and mentioned that to Strömgren. "Oh yes," he said, "that is a very good one, but beware of the mis-prints in his Comptes Rendus publication! They are not so easy to see!" Strömgren kept himself updated, so much that he was used by numerous of us more lazy readers as a living advisory encyclopedia. His encyclopedic 
knowledge was also evident at seminars and meetings when he would sit in the middle of the front row and always ask a question or make a comment that would enlighten the audience on the real importance of the work being presented. Another characteristic was his willingness to engaging himself in seemingly routine aspects of a project, such as calculating tables, reducing photometric observations or plotting data. He presumably wanted to make sure that everything was correctly done, and digested the information in the data by working on them. I also had the impression that this "handicraft" gave him a certain pleasure in itself, maybe a certain kind of calm that you experience when you do more or less difficult things that you are used to do and master since very long.

In approaching his scientific problems, Strömgren was continuously combining astrophysics and dynamics, developing his already deep insights into most fields of astronomy and exercising his methodological skills. His unusual mastery of all sorts of frontier methodology, from complex numerical calculation to the design of new optical systems, from astrometry to detailed spectroscopy, from quantum-mechanical calculations to reducing observations, from lining up observing programs to making model atmospheres and analysing stellar abundances, is of course not easy to match today. Our mode of operation, in research teams with different specialities represented by different individuals, may be necessary. But Strömgren's mode had its virtues; in particular, it made it possible for him to answer his questions in the right order, starting by defining the problem. He could design his research optimally and follow the problem wherever it brought him methodologically. Maybe we should attempt learning more methods throughout life, and not allow ourselves to become specialists in only one narrow field.

Simultaneously with this keen interest in details, Strömgren was also a grand visionary. Those visions were, however, almost obvious to him. In his autobiographical notes (Strömgren 1983) he states in the end, after having listed a number of planned applications of his new methods: "The connection of the various research goals just mentioned with broader areas of investigation - Galactic spiral structure, star formation, early phases of Galactic evolution - is clear, and I shall not comment on it here." When looking back at his work, however, it is also clear that he succeeded in moving through his interesting landscape of details, with lots of interest and care of these details, without losing track. It is clear from some of his reviews, as well as from his popular articles, that he was what Zygmunt Baumann has called a pilgrim, systematically travelling towards a goal beyond the horizon but on the way cultivating himself, his pupils, and his surroundings in an engaged way (Baumann 1993).

Another aspect gets also very clear when reading his papers. He was very much an empiricist, in spite of his extraordinary theoretical skills. His approach towards Galactic evolution was similar to that of a true historian of nature, or even an archaeologist. In discussing Nobel Prize candidates once, he said: "One should always ask oneself the question whether the scientific world would have been any different if this person had not lived." To him, this pointed more towards people who had done innovative empirical work than those who had come up with fancy speculations first. Incidentally, Strömgren lived up to this criterion; astronomy would have been different and less interesting if he had not lived.

Yet, another characteristic of Strömgren, and his way of working, was his interest in other people, not the least young scientists, and their work. He contributed important inspiration to younger colleagues in Denmark and elsewhere to develop their own specialities and gave them their place in his grand vision, from the astrometrical work of Erik $\mathrm{H} ø \mathrm{~g}$ and Lennart Lindegren, leading to the Hipparcos and Gaia satellites to the breakthrough at Nordita and Copenhagen Observatory as regards simulation of convection in stars and the Sun by Åke Nordlund. He once had an appointment with the director at 
the HC Ørsted Institute, on the other side of the Fælled Park, and I accompanied him there in order to visit the place. He suggested that we should enter a side door of the building, and we came into the workshop, where he immediately engaged in discussion with an engineer. We next proceeded to another floor where he soon was discussing thesis projects with graduate students that he had not met before. During the final, and rather late, approach towards the office of the Director of the Institute, he remarked to me: "It is a good idea to talk to the young fellows first. You tend to learn more from them." In this way, many young scientists have been encouraged by Strömgren's interest and benefited from his advice.

Finally, one could say that Strömgren showed great skill in "being at the right place at the right time", being in Copenhagen during the grand era of Quantum Mechanics, in Chicago and Yerkes Observatory with Otto Struve, Chandrasekhar and W.W. Morgan, being in the Nordic area and in touch with Bertil Lindblad (who served as President of the IAU when Strömgren was Secretary General) who could point out the previous Swedish work on low-resolution spectrophotometric criteria when Strömgren contemplated his photometric system, and being at The Center for Advanced Study at Princeton during its golden age in the fifties and sixties. Maybe one could also say that returning to Europe when ESO developed, and to Denmark and a team of young Danes on long-term contracts, willing to undertake major, systematic studies under his supervision at a time when his programme had reached a stage when such studies were needed, was also a very wise move.

However, this is certainly an argument in retrospect. Maybe, he would under quite different circumstances still have formed an impressive programme which would have been consistent and logical. Also, the very idea that he, in his various environments, was exposed to various expertise, scientific and technological developments, is perhaps not the most relevant aspect. He was so scientifically curious and interested that he found people with new good ideas and interacted with them. Then, his extraordinary ability to combine and integrate new patterns of these various ideas, thoughts, methods, facts, into a wholeness made these interactions rewarding for all who met him. We could bring home a message from this, a message which almost seems to be inscribed in the title of his autobiographical notes (Strömgren 1983), Scientists I have known and some astronomical problems I have met: If you are open-minded and generous when meeting scientists so you learn to know them and their worlds, you also meet good problems.

\section{Final words}

Within my limited space and time there has been no possibility to properly review Strömgren's very significant contributions on the organisational level, for Danish astronomy and science in general, as well as for European and international astronomy at large. But in one of his administrative functions, that of Director of Yerkes Observatory in the middle of last century, he gave a speech on the development of astrophysics during the first half of the 20th century, at the 50th anniversary of the Observatory (Strömgren 1951b). He cited Simon Newcomb who, at the dedication of the observatory in 1897, had said: "Slow indeed is progress in the solution of the greatest problems, when measured by what we want to know. Some questions may require centuries, others thousands of years for their answer. And yet never was progress more rapid than during our time. In some directions our astronomers of today are out or sight of those of fifty years ago; we are even gaining heights which, twenty years ago, looked hopeless. Never before had the astronomer so much work, good, hard, yet hopeful work before him as today." 
"Another 50 years have passed", said Strömgren, "but Newcomb's comment is as fresh and pertinent today as it was when the Yerkes Observatory first opened its doors." Well, Strömgren and others have, not only literally spoken, opened doors to advanced science to us. We have just to be thankful, enjoy it, and work hard.

\section{References}

Baade, W. 1944, The resolution of Messier 32, NGC 205, and the central region of the Andromeda nebula, $A p J, 100,137$

Barbier, D. \& Chalonge, D. 1939, Remarques préliminaires sur quelques propriétés de la discontinuité de Balmer dans les spectres stellaires, Ann. Ap., 2, 254

Baschek, B. 1960, Abhängigkeit des Strömgrenschen Index m und der Farbindexen U-B, B-V von der Metallhäufigkeit bei sonnenähnlichen Sternen, Z. Astrophys, 50, 296

Baumann, Z. 1993, Postmodern ethics, Cambridge, MA

Blaauw, A. 1995, Stellar evolution and the population concept after 1950; The Vatican conference, Stellar populations. Proc IAU Symp 164, eds. P.C. van der Kruit, G. Gilmore, p. 39

Chamberlain, J. W. \& Aller, L. H. 1951, The atmospheres of A-type subdwarfs and 95 Leonis, ApJ, 114, 52

Conti, P. S. \& Deutsch, A. J. 1966, Color anomalies and metal deficiencies in solar-type diskpopulation stars, ApJ, 145, 742

Contopoulos, G. \& Strömgren, B. 1965, Tables of plane Galactic orbits, Publ. Inst. Space Studies, Goddard Space Flight Center, NASA

Gilmore, G. \& Reid, N. 1983, New light on faint stars. III - Galactic structure towards the South Pole and the Galactic thick disk, MNRAS, 202, 1025

Johnson, H. L. \& Morgan, W. W. 1951, On the colour-magnitude diagram of the Pleiades, ApJ, 114,522

Kelsall, T. \& Strömgren, B. 1965, Calibration of the Hertzsprung-Russell diagram in terms of age and mass for main-sequence B and A stars, Vistas in Astronomy, 8, 159

Lindblad, B. 1922, Spectrophotometric methods for determining stellar luminosity, ApJ, 55, 85

Lindblad, B. 1925, 1926, Uppsala Medd. No, 3, 4, 6, 13

Lindblad, B. 1927, On the state of motion of the Galactic system, MNRAS, 87, 553

Lindblad, B. \& Stenquist, E. 1934, On the spectrophotometric criteria of stellar luminosity, Stockholm Obs. Astr. R., 11, No. 12

Murphy, S., Keller, S., \& Schmidt, B., et al. 2008, SkyMapper and the Southern Sky Survey; a valuable resource for stellar astrophysics, ASP Conf. Ser., XXX, YYY In press, presently http://fr.arxiv.org/abs/0806.1770

O'Connell, D. J. K. 1958, ed., Stellar Populations. Proceedings of the Conference sponsored by the Pontifical Academy of Science and the Vatican Observatory, North Holland Publ. Co., Amsterdam, Interscience Publishers, Inc., New York

Öhman, Y. 1935, Some preliminary results from a study of hydrogen absorption for stars in $h$ and $\chi$ Persei, Stockholm Obs. Ann., 12, 1

Oort, J. H. 1926, The stars of high velocity, Groningen Pub., 40, 1

Oort, J. H. 1958, Dynamics and evolution of the Galaxy, in so far as relevant to the problem of the populations, in Stellar Populations. Proceedings of the Conference sponsored by the Pontifical Academy of Science and the Vatican Observatory, ed. D.J.K. O'Connell, p. 415

Petrie, R. M. \& Maunsell, C. D. 1949, The Stark effect in hydrogen as a criterion of luminosity in the early A-type stars, PASP, 61, 158

Rebsdorf, S. O. 2005, The Father, the Son and the Stars. Bengt Strömgren and the History of Twentieth Century Astronomy in Denmark and in the USA, The Steno Institute, University of Århus

Roman, N. G. 1954, A group of high velocity F-type stars, AJ, 59, 507

Stebbins, J. \& Whitford, A. E. 1943, Six-color photometry of stars I. The law of space reddening from the colors of $\mathrm{O}$ and B stars, ApJ, 98, 20

Strömgren, B. 1932, The opacity of stellar matter and the hydrogen content of the stars, Z.f. Astrophys., 7, 222 
Strömgren, B. 1937, Aufgaben und Probleme der Astrophotometrie, and Objektive photometrische Methoden, in Handbuch der Experimentalphysik,26, p. 321 and 797

Strömgren, B. 1939, The physical state of interstellar hydrogen, ApJ, 89, 526

Strömgren, B. 1940, On the chemical composition of the solar atmosphere, Festschrift für Elis Strömgren. Astronomical papers dedicated to Elis Strömgren, Einar Munksgaard, Copenhagen, p. 218

Strömgren, B. 1950, Spectrophotometry of stars with interference filters, IAU Trans, VII, p. 404

Strömgren, B. 1951a, Spectral classification through photoelectric photometry with interference filters, $A J, 56,142$

Strömgren, B. 1951b, On the development of astrophysics during the last half century, in Proceedings of a topical symposium, commemorating the 50th anniversary of the Yerkes Observatory and half a century of progress in astrophysics, McGraw-Hill, New York, ed. J.A. Hynek, p. 1

Strömgren, B. 1954, Spectral classification through photoelectric photometry in narrow wavelength regions, $A J, 59,193$

Strömgren, B. 1955, Introductory remarks: Stellar atmospheres and line profiles, in Proc. of the NSF conference on Stellar Atmospheres, ed. Marshal H. Wrubel, p. 90

Strömgren, B. \& Gyldenkerne, K. 1955, Spectral classification of G and K stars through photoelectic photometry with interference filters, ApJ, 121, 43

Strömgren, B. 1958a, Composition differences between stellar populations, in Stellar Populations. Proceedings of the Conference sponsored by the Pontifical Academy of Science and the Vatican Observatory, ed. D.J.K. O'Connell, p. 245

Strömgren, B. 1958b, Spectrophotometric classification of the population groups, in Stellar Populations. Proceedings of the Conference sponsored by the Pontifical Academy of Science and the Vatican Observatory, ed. D.J.K. O'Connell, p. 385

Strömgren, B. 1958c, The composition of stars and their ages, Observatory, 78, 137

Strömgren, B. 1963a, Problems of internal constitution and kinematics of main sequence stars, Quarterly Journal of the RAS, 4, 8

Strömgren, B. 1963b, Quantitative classification methods, in Basic Astronomical Data: Stars and stellar systems, ed. K.A. Strand, University of Chicago Press, Chicago, p. 123

Strömgren, B. 1966a, Age determination for main-sequence B, A, and F stars, in Stellar evolution. Proceedings of an international conference, Nov. 13-15, 1963, sponsored by the Institute for Space Studies of the Goddard Space Flight Center, NASA, eds. R.F. Stein, A.G.W. Cameron, Plenum Press, New York, p. 391

Strömgren, B. 1966, Spectral classification through photoelectic narrow-band photometry, Ann. Rev. $A \& A, 4,433$

Strömgren, B. 1967, Places of formation of young and moderately young stars, IAU Symp., 31, 323

Strömgren, B. 1969, Quantitative Spektralklassifikation und ihre Anwendung auf Probleme der Entwicklung der Sterne und der Milschstrasse (Karl-Schwarzschild-Vorlesung), Mitt. Astron. Ges., 27, 15

Strömgren, B. 1972, Interstellar reddening within 200 pc of the Sun, Quarterly Journal of the RAS, 13,153

Strömgren, B., Olsen, E. H., \& Gustafsson, B. 1982, Evidence of helium abundance differences between Hyades stars and Coma cluster stars, PASP, 94, 5

Strömgren, B. 1983a, Scientists I have known and some astronomical problems I have met, Ann. Rev. A\&SA, 11, p. 1

Strömgren, B. 1985, Star counts, local density and K(z) force, IAU Symp., 106, 153

Strömgren, B. 1987, An investigation of the relations between age, chemical composition and parameters of velocity distribution based on uvby $\beta$ photometry of $\mathrm{F}$ stars within $100 \mathrm{pc}$, The Galaxy, eds. G. Gilmore and B. Carswell, D. Reidel Publ. Co., p. 229 